\title{
Human subcutaneous dirofilariasis: an increasing phenomenon in Sri Lanka
}

\author{
A.H.M.G.B. Abeysinghe, R.A.M. Jiffry, W.M.M. Perera \\ District General Hospital, Matale, Sri Lanka.
}

Key words: Dirofilariasis; subcutaneous; endemic

\begin{abstract}
Dirofilariasis, an emerging zoonosis around the world is endemic in Sri Lanka. Dirofilaria repens, manifesting as subcutaneous and ocular disease is the most commonly reported species. In Sri Lanka, ocular dirofilariasis has been extensively reported. However, literature on subcutaneous dirofilariasis is limited. Here we report a series of cases of subcutaneous dirofilariasis encountered within a period of approximately a year, mainly among the paediatric population in the Matale district. Noting the increasing frequency of reported cases we would like to emphasize on the importance of considering subcutaneous dirofilariasis among the differential diagnosis of subcutaneous lumps and bumps.
\end{abstract}

\section{Introduction}

The dirofilarial zoonosis caused by the nematode is mainly found among domestic dogs, and to some extent among cats and other wild canines which act as the definitive hosts and reservoir of the disease. Humans are inadvertent dead-end hosts, transmitted by the mosquito vectors during a blood meal. The first documented report of human dirofilariasis dates back to the report of Addario in 1885 from Italy. In Sri Lanka, the zoonosis is endemic [1]. And the first human case was reported in 1962 [2]. The emerging zoonosis is being reported in increasing numbers around the world [3]. Among the 40 recognized species, Dirofilaria repens, Dirofilaria ursi, Dirofilaria tenuis and Dirofilaria striata are found in the subcutaneous tissues.

\section{Case presentation}

One, 2,8,10 and 32 year old patients presented with subcutaneous lumps in the left forearm, right upper calf, right scalp, left parietal region and right breast respectively for a duration ranging from several days to 4 months. They ranged in size between $8 \times 3 \mathrm{~mm}$ and $1 \times 1.5 \mathrm{~cm}$. There were no

Correspondence: R.A.M. Jiffry

E-mail: rishadjiffry@gmail.com

Received: 22-01-2018 Accepted: 04-02-2018

(iD) http://orcid.org/0000-0002-3361-3108

DOI: http://doi.org/10.4038/sljs.v36i1.8480

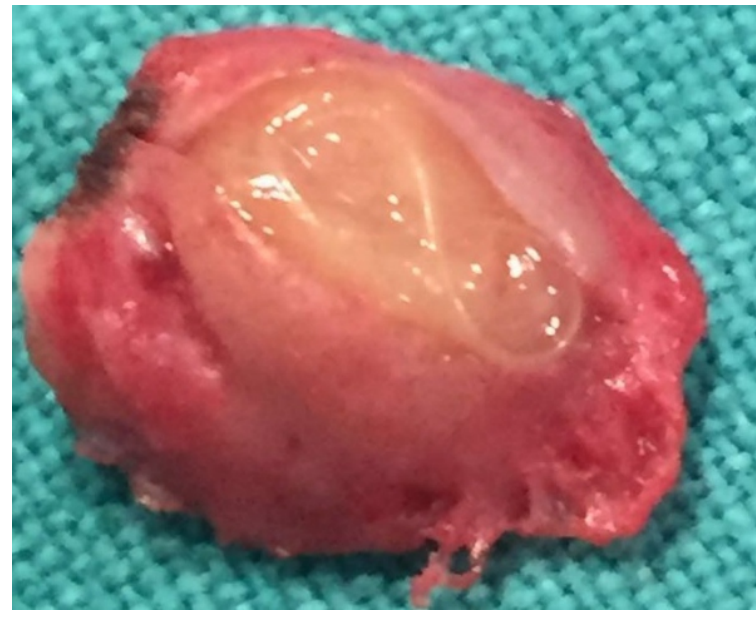

Figure 1. Subcutaneous lump

associated local inflammatory features or systemic manifestations. Ultrasonography in all cases revealed a hypoechogenic subcutaneous mass with a linear echogenic structure inside, suggestive of a worm granuloma. CBC parameters were within normal limits with a normal eosinophil count. Subsequent surgical excision was done. The histopathological examination of the wall revealed heavy infiltrate of eosinophils, suggestive of a parasitic infestation and parasitological analysis identified dirofilarial species.

\section{Discussion}

The human dirofilariasis is a parasitic infection caused by the helminth belonging to the genus dirofilaria, with the species D. repens and D. immitis being the most widespread form in the world [4]. Our extensive search of the literature revealed that no cases of D. immitis have been reported in Sri Lanka. It is a common zoonotic disease among canines. The vector born disease is dependent upon arthropods - the intermediate hosts, mainly mosquitoes belonging to the genera Aedes aegypti, Armigeres subalbatus, Mansonia uniformis and Mansonia annulifera in Sri Lanka, but also fleas, lice and ticks to transmit the disease to the definitive hosts [5]. Adult female worms in the definitive hosts produce microfilariae (L1), ingested by the arthropod during a blood meal. It develops through to an infective third stage larva (L3) in the intermediate host, finally reaching the salivary glands, allowing transmission to a new host during a subsequent blood meal. 
Humans, although infected in the same way, are accidental dead-end hosts and the worms do not reach maturity in the subcutaneous tissues and therefore do not become microfilaremic. However one case of circulating microfilaremia has been reported in Corsica [6]. The presence of the nematode in the subcutaneous tissue gives rise to a chronic inflammatory reaction, resulting in the formation of a worm granuloma as evidenced by the histopathologic findings in our cases.

As the parasitic development ceases at temperatures below $14^{\circ} \mathrm{C}$ the disease occurrence is more prominent in countries with warmer climate; this is also a possible reason behind the increasing frequency of cases in keeping with the global climate changes. Several other factors may be responsible for the increasing incidence, including greater access to medical and diagnostic facilities. It is endemic in the Mediterranean basin, with the highest prevalence being reported in Italy among the European countries, and Sri Lanka in Asia. Although the infection with these helminths is independent of dog ownership, residence or travel to areas where canine dirofilariasis is endemic is universal among the reported human cases. The rate of dirofilariasis in dogs in Sri Lanka is said to be as high as $30-60 \%$ [5]. The mosquito vectors, feeding indiscriminately on different animal species and humans further increases the risk of infection in the endemic areas.

Globally, it is considered to be more common in adults, peaking among the age group of 40 to 49 years, and in the upper body sites (eyes and the face accounting for up to $46 \%$ ) [3]. However among the Sri Lankan population it is more common in children, under the age of 9 years, in agreement with the findings among our cases [5]. It is also said to be localized to the lower body regions in Sri Lanka.

The usual presentation is with asymptomatic subcutaneous nodules, although rare cases such as meningoencephalitis and aphasia and acute abdomen and peritonitis due to intraabdominal infection have been reported $[7,8]$. Conforming to this our patients did not complain of any pain, itching or redness. Although peripheral eosinophilia is said to be observed in up to $20 \%$ cases of human dirofilariasis, none of our cases showed results supportive of this in CBC analysis. Ultrasonography was used to aid in diagnosis, whereas imaging modalities such as computed tomography scan (CT) and Magnetic resonance imaging (MRI) were considered inessential given the nature of presentation. As humans are dead end hosts, surgical excision is considered to be the standard of treatment. The diagnosis of dirofilariasis can then be made largely based on the morphological features of the worm [4]. However, polymerase chain reaction analysis would aid not only in quantification but also precise identification and differentiation of species. Identification based on the morphological features has its pitfalls, considering similarities between species and alterations secondary to inflammatory response or surgical artefact $[7,9]$.

\section{Conclusion}

It is essential to keep in mind human subcutaneous dirofilariasis as a differential for subcutaneous lesions. In the meantime adequate measures for vector control has to be initiated.

All authors disclose no conflict of interest. The study was conducted in accordance with the ethical standards of the relevant institutional or national ethics committee and the Helsinki Declaration of 1975, as revised in 2000 .

\section{References}

1. Nath R, Gogoi R, Bordoloi N, Gogoi T. Ocular dirofilariasis. Indian J Pathol Microbiol. 2010;53:157-9. https://doi.org/10.4103/0377-4929.59213

2. S.E. Wijetilake, D. Attylgalle, A.S. Dissanaike.

A case study of human infection with Dirofilaria repens. Clinical presentation and aspects of transmission. Proc Kandy Soc Med, 9(1962), pp. 23-24

3. Pampiglione S, Rivasi F.

Human dirofilariasis due to Dirofilaria (Nochtiella) repens: An update of world literature from 1995 to 2000. Parassitologia. 2000;42:231-54.

4. F. Simón, M. Siles-Lucas, R. Morchan, J. González-Miguel, I. Mellado, E. Carretón, et al.

Human and animal dirofilariasis: the emergence of a zoonotic mosaic. Clin Microbiol Rev, 25 (2012), pp. 507-544.

https://doi.org/10.1128/cmr.00012-12

5. A.S. Dissanaike, W. Abeyewickreme, M.D.Wijesundera, M.V. Weerasooriya, M.M. Ismail.

Human dirofilariasis caused by Dirofilaria (Nochtiella) repens in Sri Lanka. Parassitologia, 39 (1997), pp. 375-382

6. Nozais JP, Bain O, Gentilini M. A case of subcutaneousdirofilaria (Nochtiella) repens with microfilaremia originating in Corsica. Bull Soc Pathol Exot. 1994. 87(3):183-5

7. Poppert S, Hodapp M, Krueger A, Hegasy G, Niesen WD, Kern $\mathrm{WV}$, et al.Dirofilaria repens infection and concomitant meningoencephalitis. Emerg Infect Dis. 2009;15:1844-6. https://doi.org/10.3201/eid1511.090936

8. Revesz E, Markovics G, Darabos Z, Toth I, Fok E. Dirofilaria in the abdominal cavity. Magy Seb. 2008 Oct. 61(5):281-4. https://doi.org/10.1556/MaSeb.61.2008.5.6.

9. Pampiglione S1, Rivasi F, Canestri-Trotti G. Pitfalls and difficulties in histological diagnosis of human dirofilariasis due to Dirofilaria (Nochtiella) repens. Diagn Microbiol Infect Dis. 1999 May;34(1):57-64. https://doi.org/10.1016/s0732-8893(98)00164-3 